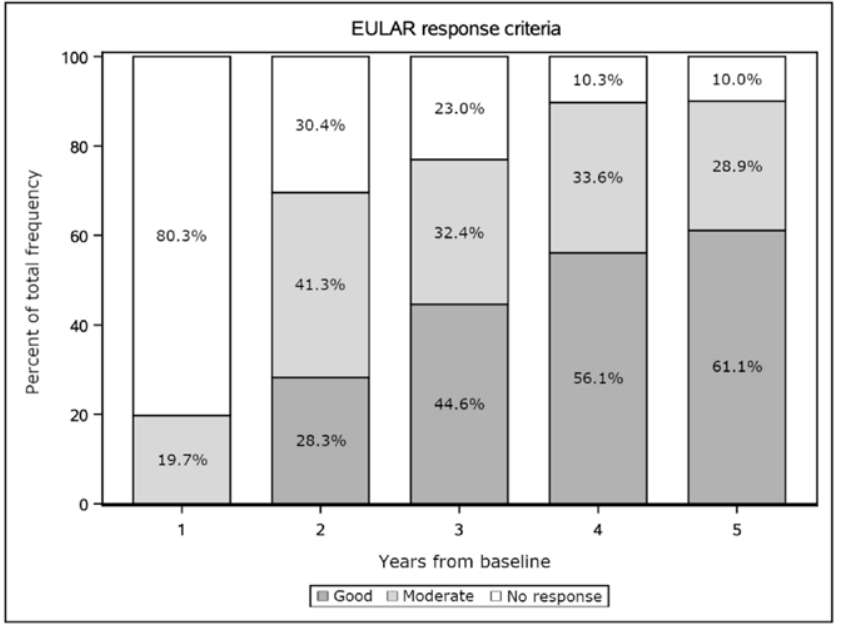

Figure 1. No, moderate and good response to RTX treatment

No significant difference during the 5-year follow-up was found regarding previous use of bDMARD or not and for concomitant use of csDMARDs or not for variables listed in the table 1.

Drug survival for the RTX was $83 \%(95 \mathrm{Cl} 77-87 \%)$ after 1 year, $66 \%(95 \mathrm{Cl}$ $60-72 \%)$ after 2 years, $53 \%(95 \mathrm{Cl} 46-59 \%)$ after 3 years, $46 \%(95 \mathrm{Cl} 39-52 \%)$ after 4 years and $34 \%(95 \mathrm{Cl} 28-40 \%)$ after 5 years of follow up.

No significant difference in drug survival was found between bDMARD naïve and previous users of bDMARDs or between concomitant and non-concomitant users of csDMARDs. RF positive patients had a better drug survival.

In prediction analysis RF positive status, high baseline DAS28, low baseline CRP, previous bDMARDs use, short disease duration and low MHAQ were found to be independently associated with better drug survival.

Conclusion: Our real life data shows that RTX treated RA patients had a satisfactory treatment response and drug survival declines rather linearly over time. However, a significant treatment response was achieved primary in the second year indicating that at least 2 twin infusions should be given before identifying treatment failure.

REFERENCES:

[1] Mok CC. Rituximab for the treatment of rheumatoid arthritis: An update. Drug Des Devel Ther 2013;8:87-100.

[2] Fransen J, van Riel PL. The Disease Activity Score and the EULAR response criteria. Rheum Dis Clin North Am. 2009;35:745-viii.

Acknowledgements: Nurses and doctors from Division of Rheumatology at Sørlandet Hospital in Kristiansand, Norway contributed to data collection. Disclosure of Interests: None declared DOI: 10.1136/annrheumdis-2021-eular.1661

\section{AB0220 ANTI-CITRULLINATED PROTEIN ANTIBODY (ACPA) POSITIVITY IS ASSOCIATED WITH REDUCED WITHDRAWAL RATES OF ABATACEPT IN RHEUMATOID ARTHRITIS BUT ONLY IN PATIENTS WHO ARE ANTI-NUCLEAR ANTIBODY (ANA) NEGATIVE}

J. Kimpton ${ }^{1}$, M. Shipa ${ }^{2}$, E. Hawkins ${ }^{2}$, S. A. Yeoh ${ }^{2}$, M. Greenwood ${ }^{3}$, M. Ehrenstein ${ }^{2} .{ }^{1}$ University College London, Centre for Clinical Pharmacology and Therapeutics, London, United Kingdom; ${ }^{2}$ University College London, Centre for Rheumatology, London, United Kingdom; ${ }^{3}$ University College London Hospital, Pharmacy Department, London, United Kingdom

Background: Abatacept, a selective inhibitor of T cell co-stimulation, is often used as a second-line biologic disease-modifying anti-rheumatic drug (bDMARD) after the failure of tumour necrosis factor inhibitor (TNFi) in Rheumatoid Arthritis (RA). However, in comparison to TNFi very few survival analyses of abatacept have been reported. ${ }^{1,2}$

Objectives: To investigate predictors of abatacept discontinuation due to either inefficacy or adverse events in RA patients over 5-years.

Methods: A retrospective observational analysis was conducted on a tertiary hospital dataset of RA (according to 2010 ACR/EULAR criteria) patients who started abatacept (either intravenous or subcutaneous). Time to abatacept discontinuation over 5-years was estimated using Kaplan-Meier survival analyses. A multivariate cox-regression model to predict abatacept discontinuation was chosen by elastic net regularisation.
Results: A total of 112 patients with RA [81\% female, mean age 58.1 (SD 13.5) years] commenced abatacept therapy during the study period. 88 (78.6\%) patients received intravenous abatacept, $14(15.9 \%)$ of whom switched to subcutaneous injection, and $24(21.4 \%)$ were initially treated with subcutaneous abatacept, $2(8.3 \%)$ of whom switched to intravenous. More than half of the patients $(65 / 112)$ were treated with at least one concomitant conventional synthetic DMARD (csDMARD). Methotrexate was the most commonly used $(n=37)$ csDMARD, followed by hydroxychloroquine $(n=23)$, sulfasalazine $(n=15)$, and leflunomide $(n=7) .42(37.5 \%)$ patients were treated with glucocorticoids (either oral, intra-articular, or intramuscular injection) during the time they were treated with abatacept. Abatacept was most commonly used as 4th $(n=29)$ and 3rd line $(n=24)$ bDMARD but 19 patients received abatacept as their first line bDMARD. $75(67 \%)$ patients were rheumatoid factor (RF) positive and $73(65.2 \%)$ were anti-citrullinated protein antibody (ACPA) positive. Anti-nuclear antibody (ANA) was positive in 32 patients. Abatacept was discontinued in 54 patients (48.2\%); $19(35.2 \%)$ due to an adverse event and 35 (64.8\%) due to loss of efficacy. Overall, the median time to discontinuation of abatacept was 3.8 years.

Multivariate cox regression (variables chosen by the elastic net and adjusted by whether or not abatacept was used as first-line therapy) showed that ACPA positivity was associated with a reduced risk of abatacept discontinuation with a hazard ratio (HR) of $0.40(95 \% \mathrm{Cl} 0.18$ to $0.85, \mathrm{p}=0.02, \mathrm{~N}$. of events $16 / 47)$ compared to ACPA negative patients (N. of events 15/23), but only if ANA was negative. In contrast, ACPA positivity did not reveal any retention benefit over ACPA negative patients, if they were ANA positive.

ACPA positive patients without positive ANA increased the time-to-discontinuation of abatacept predominantly after 3 -months (unadjusted log-rank $p=0.02$ ), compared to ACPA, and ANA negative patients (Figure 1). Adding csDMARDs with abatacept, reduced the risk of discontinuation of abatacept by $59 \%(95 \%$ $\mathrm{Cl} 24 \%$ to $77 \%, \mathrm{p}=0.004, \mathrm{~N}$. of events $26 / 65$ ) compared to monotherapy (N. of events 28/47).

Conclusion: Our data suggests patients who are ACPA positive and ANA negative are more likely to remain on abatacept therapy. Concomitant csDMARD use also acts as a positive predictor of abatacept treatment retention.

\section{REFERENCES:}

[1] Cagnotto G, et al. Arthritis Res Ther. 2020;22(1):15

[2] Alten R, et al. Clin Rheumatol. 2019;38(5):1413-1424.

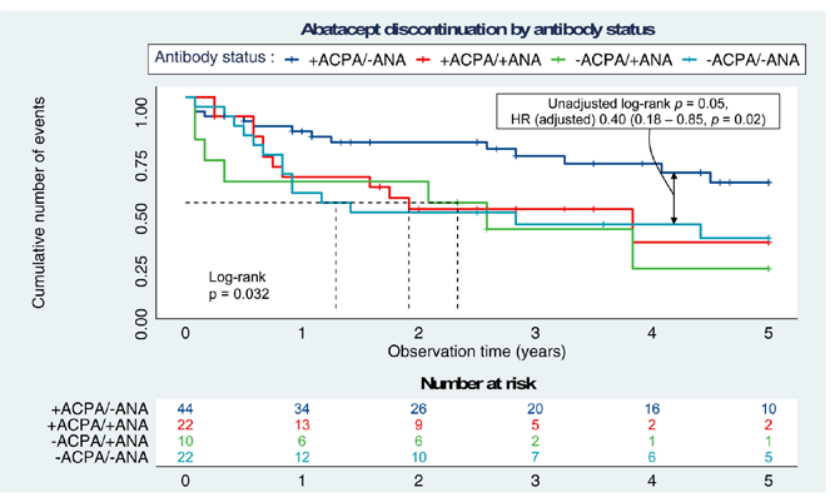

Figure 1. Kaplan-Meier survival curve of retention of abatacept, stratified by Anti-citrullinated protein antibody (ACPA) and antinuclear antibody (ANA).

$+A C P A /-A N A=A C P A$ positive and ANA negative, $+A C P A /+A N A=A C P A$ positive and $A N A$ positive, $-A C P A /-A N A=A C P A$ negative and $A N A$ negative, $-A C P A /+A N A=A C P A$ negative and $A N A$ positive, $H R=$ Hazard ratio.

Acknowledgements: I have no acknowledgements to declare.

Disclosure of Interests: None declared

DOI: 10.1136/annrheumdis-2021-eular.1848

\section{\begin{tabular}{|l|l}
\hline AB0221 CURRENT IMPLEMENTATION OF TREAT-TO-TARGET \\
\hline
\end{tabular} APPROACH IN RHEUMATOID ARTHRITIS TREATMENT: THE PERSPECTIVE OF CHINESE RHEUMATOLOGISTS}

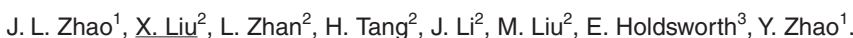
${ }^{1}$ Peking Union Medical College Hospital, Department of Rheumatology, Beijing, China; ${ }^{2}$ Eli Lilly and Company, Medical, Shanghai, China; ${ }^{3}$ Adelphi Real World, Rheumatology, Manchester, United Kingdom

Background: Treat-to-target (T2T) approach is recommended as a standard management strategy in rheumatoid arthritis (RA) treatment by Chinese guideline for diagnosis and treatment of $\mathrm{RA}^{[1]}$. However, there is little known about its current implementation in China. 
Objectives: This study aimed to evaluate the implementation and achievement of T2T approach and explore their associated factors in Chinese RA cohort. Methods: A comprehensive cross-sectional survey of rheumatologists and their RA patients was conducted in China. Data were collected during May-Aug 2019 via physician-completed patient record forms. 60 rheumatologists provided data on demographic, clinical characteristics, treatments, and T2T approach implementation for 600 RA patients. Two logistic regressions were used to evaluate factors associated with T2T approach implementation and T2T goal achievement, respectively. Patients with missing data were not included in the models. Results: 600 patients were included in this study $(48.8 \pm 11.7$ years, $70.3 \%$ female). $39.0 \%(\mathrm{~N}=234)$ of 600 patients were being treated with T2T approach, and $64.9 \%(\mathrm{~N}=366)$ of 564 patients had achieved T2T goal. Patients with longer disease duration (>2 years diagnosis) (odds ratio $(\mathrm{OR})[95 \% \mathrm{Cl}]=1.61[1.05,2.49]$, vs. diagnosis $\leq 2$ years), higher pain score (OR $[95 \% \mathrm{Cl}]=1.26[1.04,1.51])$, or receiving advanced therapy $(\mathrm{OR}[95 \% \mathrm{Cl}]=6.91[3.64,13.13])$ were more likely to use T2T. Patients with $\mathrm{BMI}>23.9 \mathrm{~kg} / \mathrm{m}^{2}$ (OR $[95 \% \mathrm{Cl}]=2.83[1.59,5.04]$, vs. $\left.\mathrm{BMI} \leq 23.9 \mathrm{~kg} / \mathrm{m}^{2}\right)$, or who worked full-time $(\mathrm{OR}[95 \% \mathrm{Cl}]=2.12[1.26,3.57])$ were more likely to achieve T2T goal, while patients with more pain $(\mathrm{OR}[95 \% \mathrm{Cl}]=0.77$ $[0.64,0.92])$ were less likely to achieve T2T goal.

Conclusion: Low implementation of T2T approach is observed in Chinese RA treatment. Longer disease duration, more pain, and receiving advanced therapy are associated with higher probability of T2T use, while higher BMI, full-time work and less pain are associated with higher probability of T2T goal achievement. Standard diagnosis and treatment according to guidelines may improve T2T approach implementation.

\section{REFERENCES:}

[1] Association, C.R., 2018 Chinese guideline for the diagnosis and treatment of rheumatoid arthritis. Zhonghua nei ke za zhi, 2018. 57(4): p. 242.

Disclosure of Interests: Jiu-liang Zhao: None declared, Xin Liu Employee of: Eli Lilly and Company, Lujing Zhan Employee of: Eli Lilly and Company, Hongyu Tang Employee of: Eli Lilly and Company (Intern), Jinnan Li Employee of: Eli Lilly and Company, Mengru Liu Employee of: Eli Lilly and Company, Elizabeth Holdsworth Consultant of: Eli Lilly and Company, Employee of: Adelphi Real World, Yan Zhao: None declared

DOI: 10.1136/annrheumdis-2021-eular.1960

\section{AB0222 2 COMPARISON OF SELF-INJECTION DEVICES FOR ADMINISTERING ANTI-TUMOR NECROSIS FACTOR AGENTS USING THE ORIGINAL QUESTIONNAIRE IN PATIENTS WITH RHEUMATOID ARTHRITIS}

Y. Hirano ${ }^{1}$, J. Hasegawa ${ }^{1} .{ }^{1}$ Toyohashi Municipal Hospital, Rheumatology, Toyohashi, Japan

Background: Self-injection of biological agents has become a general practice in the treatment of rheumatoid arthritis (RA). Self-injectable biological agents differ in shape, needle thickness, drug fluid volume, and so on. These differences may affect patients' evaluations of self-injection devices. Five kinds of anti-tumor necrosis factor (TNF) agents can be administered by self-injection in clinical practice in Japan. Information on patients' evaluations of self-injection devices is important in choosing the agent for the treatment of RA among several agents with the same mechanism of action.

Objectives: The aim of this retrospective cross-sectional study was to compare patient evaluations of anti-TNF self-injection devices in the treatment of RA.

Methods: RA patients in whom etanercept (ETN) pen $50 \mathrm{mg}$ (Embrel Pen) was switched to ETN biosimilar (BS) $50 \mathrm{mg}$ (ETN-BS [MA]), adalimumab (ADA) syringe $40 \mathrm{mg}$ (Humira syringe) was switched to ADA pen $40 \mathrm{mg}$ (Humira pen), golimumab (GLM) syringe $50 \mathrm{mg}$ (Simponi syringe) was switched to GLM autoinjector (Simponi $\mathrm{Al}$ ), and certolizumab pegol (CZP) syringe (Cimzia syringe) was switched to CZP autoclicks (Cimzia AC) were asked to answer an originally developed questionnaire (Toyohashi Self-injection Assessment Questionnaire [T-SAQ]; Table 1) before and after switching agents. T-SAQ included 18 questions. A score of 0 indicated "best" and 4 indicated "worst" for each question, with a highest possible score of 72. The patients' characteristics and T-SAQ scores before and after switching agents were investigated. A statistical analysis of the difference in T-SAQ score between before and after switching was performed using the Wilcoxon signed-rank test. A P value $<0.05$ was considered significant. Results: The patients were divided into groups according to the agents they received with switching as follows: switchers from ETN pen to ETN-BS, $n=32$; switchers from ADA syringe to ADA pen, $n=28$; switchers from GLM syringe to GLM-AI, $n=25$; and switchers from CZP syringe to CZP-AC, $n=10$. The total T-SAQ scores were as follows, respectively: ETN pen and ETN-BS, 23.5 and 19.0; ADA syringe and ADA pen, 25.8 and 14.9; GLM syringe and GLM-AI, 23.8 and 17.4; and CZP syringe and CZP-AC, 30.6 and 18.8. The total T-SAQ was significantly improved after switching to the pen devices in all the switching groups. In the switchers from ETN pen to ETN-BS, the scores for questions 5, 10, 11, 12, 16,17 , and 18 were significantly improved after switching. The total T-SAQ scores were significantly improved for questions $1,2,3,4,5,7,11,12,13,15,16$, and 17 in the switchers from ADA syringe to ADA pen, for questions 1, 2, 5, 7, 10, 16, and 17 in the switchers from GLM syringe to GLM-Al, and for questions of 1, 2, 3, 4 $5,7,11$, and 15 in the switchers from CZP syringe to GLM-AC.

Conclusion: The pen devices were favorably assessed by the RA patients in whom syringe devices were switched to pen devices for the same agent. The total T-SAQ score improved in the RA patients in whom ETN pen was switched to ETN-BS probably because the thickness of the needle was thinner in the ETN-BS than in the ETN pen. The total T-SAQ score for the ADA pen was lowest probably because the thinnest needle was used (29 gauge) and the amount of drug fluid is smallest $(0.4 \mathrm{ml})$. The total T-SAQ score for the CZP syringe was the lowest probably because the thickest needle was used ( 25 gauge).

Table 1. Toyohashi Self-injection Assessment Questionnaire (T-SAQ)

\begin{tabular}{|l|l|c|c|c|c|c|}
\hline Q & Score & 0 & 1 & 2 & 3 & 4 \\
\hline 1 & Ease to use & Excellent & Very Good & Fair & Poor & Unacceptable \\
\hline 2 & Ease of learning how to use & Very easy & easy & Fair & Difficult & Very Difficult \\
\hline 3 & Ease of abandoning & Very easy & easy & Fair & Difficult & Very Difficult \\
\hline 4 & Ease of injection & Very easy & easy & Fair & Difficult & Very Difficult \\
\hline $\mathbf{5}$ & Ease of holding the device & Excellent & Very Good & Fair & Poor & Unacceptable \\
\hline $\mathbf{6}$ & Occurrence of injection fluid leak & Never & Almost not & Fair & Sometimes & Always \\
\hline 7 & Time of injection & Very short & Short & Fair & Long & Very Long \\
\hline $\mathbf{8}$ & Burden on leisure or travel & Very small & Small & Fair & Big & Very big \\
\hline $\mathbf{9}$ & Burden on household affairs or child-rearing & Very small & Small & Fair & Big & Very big \\
\hline 10 & Burden on work (only for those working) & Very small & Small & Fair & Big & Very big \\
\hline 11 & Strain or anxiety from injection & Very small & Small & Fair & Big & Very big \\
\hline 12 & Strain or anxiety from needle & Very small & Small & Fair & Big & Very big \\
\hline 13 & Disgust with or anxiety from self-injection & Very small & Small & Fair & Big & Very big \\
\hline 14 & Needlestick accident & Never & Almost not & Sometimes & Often & Always \\
\hline 15 & External appearance & Excellent & Very Good & Fair & Poor & Unacceptable \\
\hline 16 & Pain from pricking with the needle & None & Very mild & Moderate & Severe & Very severe \\
\hline 17 & Pain during drug injection & None & Very mild & Moderate & Severe & Very severe \\
\hline 18 & Pain after injection & None & Very mild & Moderate & Severe & Very severe \\
\hline
\end{tabular}

Disclosure of Interests: None declared

DOI: 10.1136/annrheumdis-2021-eular.2252

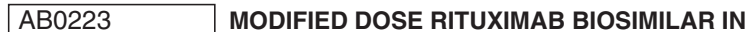 RHEUMATOID ARTHRITIS COMPARING B-CELL DEPLETION EFFECT AND DISEASE ACTIVITY SCORES}

S. Bandyopadhyay ${ }^{1} .{ }^{1}$ Apollo Gleneagles Hospital, Internal Medicine, Kolkata, India

Background: In emerging economies self-funding patients opt for less costly options, influencing both compliance and maintenance of treatment for chronic illness. Studies comparing originator rituximab 1000mgx2 and 500mgx2 doses in Rheumatoid arthritis (RA) have yielded interesting results ${ }^{1}$. Evidence of $B$ cell depletion, measured by CD19 count, maybe a marker for disease improvement ${ }^{2}$. However effect of different dose of biosimilar Rituximab (bRTX) on B cell depletion and disease activity needs exploration.

Objectives: To determine correlation of CD19 count defining B cell depletion and disease activity with different dosages of bRTX treatment.

Methods: Between April 2019 and March 2020, all RA patients with DMARD failure were screened for eligibility of biologics as routine clinical practice. Depending on individual choice, after full consent, patients received either 1000mgx2 or 500mgx 2 bRTX. All patients had CD19 count before and 12 months after the first dose. Effectiveness of bRTX $1000 \mathrm{mg} \times 2$ and $500 \mathrm{mg} \times 2$ was assessed by DAS28 and EULAR response. Comparative adjusted analysis was performed by analysis of variance (ANOVA).

Results: Out of 468 eligible patient, 84 opted for biologic. Of which 27 patients consented for bRTX (17 female, mean age 39.5 years). 13 patients opted fo $1000 \mathrm{mg} \times 2$ and 14 for $500 \mathrm{mg} \times 2$ dose. $74 \%(20 / 27)$ patients were on concomitant methotrexate and $26 \%$ on hydroxychloroquine $(7 / 27)$. Both doses led to significant reduction in ESR, CRP, and DAS28-ESR at 12 months $(p<0.001)$ (Table 1).

Table 1. RA outcome-measurement scores at 12 months post biosimilar Rituximab therapy.

\begin{tabular}{lcccc}
\hline Variable & \multicolumn{2}{c}{ Baseline } & \multicolumn{2}{c}{12 months } \\
\hline & $\begin{array}{c}\text { RTX 1000mg x } \\
2(n=13)\end{array}$ & $\begin{array}{c}\text { RTX 500mg x } \\
2(n=14)\end{array}$ & $\begin{array}{c}\text { RTX 1000mg x } \\
2(n=13)\end{array}$ & $\begin{array}{c}\text { RTX 500mg } \\
\times 2(n=14)\end{array}$ \\
\hline ESR $^{*}$ & $53.9 \pm 23.9$ & $57.1 \pm 24.7$ & $23.9 \pm 2.9$ & $24.1 \pm 4.7$ \\
CRP $^{*}$ & $6.1 \pm 3.9$ & $6.9 \pm 2.9$ & $2.1 \pm 0.9$ & $2.3 \pm 0.9$ \\
DAS28-ESR $^{*}$ & $6.1 \pm 0.3$ & $6.1 \pm 0.2$ & $4.0 \pm 0.4$ & $4.1 \pm 0.2$ \\
CD 19+ Count $^{* \#}$ & $1191.6 \pm 308.4$ & $1155 \pm 289.6$ & $128.8 \pm 90.4$ & $139 \pm 90.6$ \\
$\quad(10 / L)$ & & & & \\
\hline
\end{tabular}

$p<0.0001$ as compared to 12 mos vs baseline; ${ }^{\#} p<0.0001$ as compared amongst group 